\title{
Acute-Onset Anterograde Amnesia Caused by Isolated Bilateral Fornix Infarction
}

\author{
Philippe Rizek, Stephen Pasternak, Andrew Leung, Mary E. Jenkins
}

Can J Neurol Sci. 2013; 40: 738-739

A 56-year-old right-handed gentleman was brought to hospital by his family after noting that he had acute short-term memory difficulties within 24 hours and that he was frequently repeating the same questions every few minutes. His daughter described him as being like a tape recorder, repeating the questions "Where's my jacket? Where's my phone? Where's my watch?" continually throughout the first night of admission. His past medical history was significant for atrial fibrillation, aortic and mitral valve replacement with mechanical prostheses 11 years prior, and a right frontal stroke 11 years prior giving rise to mild disinhibition.

He had a 15 pack-year smoking history prior to his stroke, and has since only occasionally smoked cigars; he rarely consumes alcohol. He was otherwise independent with his activities of daily living. His home medications included warfarin, aspirin, ramipril, metoprolol and digoxin. There was no history of trauma or any recent systemic or constitutional symptoms. There was no family history of cerebrovascular disease or dementia. He denied any headache, vision changes, weakness, numbness, parasthesias, dysphagia, speech changes, gait difficulties, seizures or hallucinations.

On physical examination, the patient was alert and oriented; afebrile with stable vital signs. He was able to follow 3-step commands. He scored $17 / 30$ on the Montreal Objective Cognitive Assessment (MoCA), with predominant deficits in recall $(0 / 5)$ and registration $(3 / 5)$ and visuospatial/executive function (2/5) and attention (2/6). His affect was normal with mild disinhibition. There was no dysarthria or ideomotor apraxia. Primitive reflexes were absent. Reading, repetition and naming were intact, with some perseveration. He would continually repeat the same questions within one to two minutes of receiving an answer. The remainder of his neurological examination was normal, including cranial nerves II-XII, motor examination, sensory testing, reflexes, coordination, balance, and gait testing.

His blood work, including complete blood count, electrolytes, urea, creatinine, thyroid stimulating hormone, vitamin B12, liver enzymes, cardiac enzymes, inflammatory markers (erythrocyte sedimentation rate and $\mathrm{C}$-reactive protein) and toxicology screen, were within normal limits. His international normalized ratio (INR) was 2.8 (Target goal on warfarin: 2.5-3.5). His serum low-density lipoprotein level was $3.29 \mathrm{mmol} / \mathrm{L}$. Urinalysis, urine culture, and chest $\mathrm{x}$-ray were unremarkable. Electrocardiogram (ECG) demonstrated atrial fibrillation. His computed tomogram (CT) head did not demonstrate any acute lesions; the old infarct was noted in the right frontal operculum, as well as small old infarcts in the medial right occipital lobe and left cerebellum.
Magnetic resonance imaging/angiography (MRI/MRA) of the head and neck demonstrated mild fullness in the fornices bilaterally with high signal on $\mathrm{T} 2$ and diffusion sequences concerning for acute infarction; with normal appearance of intraand extracranial vessels. Transthoracic echocardiogram did not demonstrate an intracardiac thrombus, and showed normal ejection fraction (60-65\%); normal peak and gradient of the mechanical mitral valve and prosthetic aortic valves. Electroencephalogram (EEG) showed generalized grade I dysrhythmia, but no epileptiform activity. Repeat MRI with gadolinium one week later showed non-enhancing, evolving swelling and high T2 signal and diffusion restriction in the fornices bilaterally, consistent with bilateral fornix infarction (Figure).

The patient was seen in follow-up after four months. He insisted that he was fine and "all back to normal." He repeated questions and statements throughout the assessment. His MiniMental Status Exam (MMSE) was 18/30, scoring 0/3 for recall, 2/5 for spelling "World" backwards. His MoCA was 12/30, with prominent deficits in memory $(0 / 5)$, visuospatial/executive function $(2 / 5)$ and attention (1/6). He was able to register $5 / 5$ memory items. His recall was not improved by category or multiple choice cues. Because of behavioral concerns, we administered a Frontal Behavioural Inventory (FBI). The total FBI score was 21, earning 10 points for negative behaviors (e.g. inflexibility, personal neglect, loss of insight) and 11 points for disinhibition (e.g. inappropriateness, hypersexuality, irritability, restlessness). All of these personality traits were present since the stroke 11 years ago, indicating that the frontal lobe findings on the MoCA firmly pre-date the current stroke. This bilateral fornix infarction has resulted in a permanent, severe amnestic state.

\section{DISCUSSION}

The fornix is an integral part of the Papez circuit, playing a critical role in the formation of memory. Damage or disease affecting its function has been shown to result in anterograde

From the Department of Clinical Neurological Sciences (PR, SP, MEJ), Department of Medical Imaging (AL), The University of Western Ontario; Cognitive Neurology and Alzheimer's Disease Research Centre (SP), Parkwood Hospital; J. Allyn Taylor Centre for Cell Biology (SP), Molecular Brain Research Group, Robarts Research Institute, London, Ontario, Canada.

Received February 14, 2013. Final Revisions Submitted April 29, 2013. Correspondence to: Philippe Rizek, Department of Clinical Neurological Sciences, Schulich School of Medicine, The University of Western Ontario, 339 Windermere Rd, Rm B7-005, London, Ontario, N6A 5A5, Canada. Email: phil.rizek@gmail.com. 


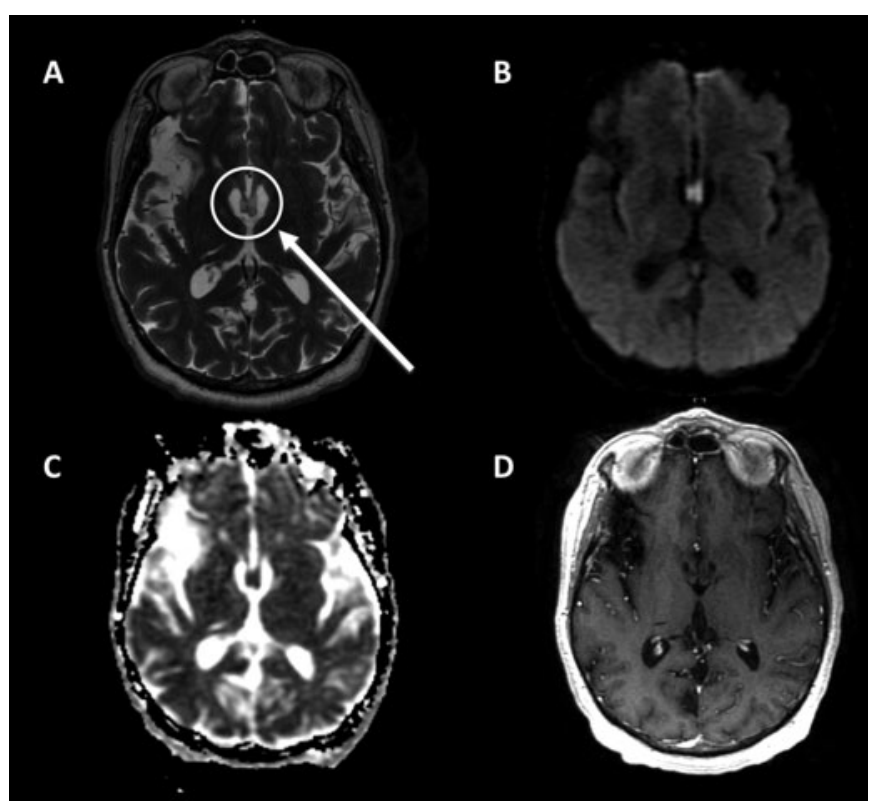

Figure: Axial MRI Head demonstrating high T2 signal and fullness in the bilateral fornices ( $A$, circle and arrow); with high signal on diffusionweighted imaging $(B)$ and low apparent diffusion coefficient $(C)$ nonenhancing with gadolinium $(D)$.

amnesia. ${ }^{1}$ Forniceal infarction with accompanying anterograde amnesia has been described after surgical repair of anterior communicating artery aneurysms. ${ }^{2}$ Non-traumatic, acute-onset anterograde memory loss due to bilateral fornix infarction is rare, with few cases reported in the literature (Table). ${ }^{3-8}$ Four of these cases also had concomitant anterior callosal infarction. ${ }^{3-6}$ This may be explained by the fact that the short branches of the anterior communicating artery, which supply the columns of the fornix, commonly supply the anterior corpus callosum, septal nuclei and anterior cingulum. ${ }^{1}$ Involvement of the genu of the corpus callosum is thought to be non-contributory to the suddenonset amnesia. ${ }^{1,5}$ Moussoutas et al. have described this entity as "amnestic syndrome of the subcallosal artery". 6
Various mechanisms have been proposed for the infarctions in these cases (Table). Our patient has multiple risk factors for stroke, including hypertension, hyperlipidemia, cigarette smoking, atrial fibrillation and valvular disease. No thrombus was identified on echocardiography and he was appropriately anticoagulated on warfarin. Neuroimaging of his intra- and extracranial vessels did not demonstrate any stenoses or thrombus. We propose the most likely etiology for his discrete isolated bilateral forniceal infarction is small vessel disease affecting small branches of the anterior communicating artery.

Until recently, there have only been two cases of isolated infarction of the bilateral anterior fornices reported, one attributed to small vessel disease ${ }^{7}$ and another case attributed to giant cell arteritis. ${ }^{8}$ We present an additional case of isolated bilateral anterior fornix infarction. This entity is likely underrecognized, and should be considered in patients presenting with acute-onset anterograde amnesia and risk factors for stroke. The striking similarity of this presentation with that of Transient Global Amnesia reinforces the idea that acute amnestic patients should be observed to ensure that their amnesia is in fact transient.

\section{REFERENCES}

1. Thomas AG, Koumellis P, Dineen RA. The fornix in health and disease: an imaging review. Radiographics. 2011;31(4):1107-21.

2. Hattingen E, Rathert J, Raabe A, et al. Diffusion tensor tracking of fornix infarction. J Neurol Neurosurg Psychiatry. 2007;78(6): 655-6.

3. Moudgil SS, Azzouz M, Al-Azzaz A, et al. Amnesia due to fornix infarction. Stroke. 2000;31(6):1418-9.

4. Park SA, Hahn JH, Kim JI, et al. Memory deficits after bilateral anterior fornix infarction. Neurology. 2000;54(6):1379-82.

5. Saito Y, Matsumura K, Shimizu T. Anterograde amnesia associated with infarction of the anterior fornix and genu of the corpus callosum. J Stroke Cerebrovasc Dis. 2006;15(4):176-7.

6. Moussouttas M, Giacino J, Papamitsakis N. Amnestic syndrome of the subcallosal artery: a novel infarct syndrome. Cerebrovasc Dis. 2005;19(6):410-4.

7. Adamovich BL, Gualberto G, Roberts $\mathrm{T}$, et al. Teaching NeuroImages: amnesia due to fornix infarction. Neurology. 2009;73(17):e86.

8. Murr N, Thaisetthawatkul P, Helvey J, et al. Selective infarction of the anterior genu fornices associated with giant cell arteritis. J Stroke Cerebrovasc Dis. 2012;21(4):327-9.

Table: Case reports of anterograde amnesia attributed to bilateral anterior forniceal infarction

\begin{tabular}{l|l|l|l}
\hline Case Reports & Patient Age, Gender & MRI findings (areas of infarction) & Proposed Etiology \\
\hline Moudgil et al., $2000^{3}$ & 71, female & B/L anterior fornices, genu of CC & Small vessel disease \\
\hline Park et al., $2000^{4}$ & 60, female & B/L anterior fornices, genu of CC & Ruptured ventricular AVM \\
\hline Saito et al., $2005^{5}$ & 71, female & B/L anterior fornices, genu of CC & Small vessel disease \\
\hline Moussouttas et al., $2005^{6}$ & 61, male & $\begin{array}{l}\text { B/L anterior fornices, septum } \\
\text { pellucidum, genu of CC, and left } \\
\text { frontal subcortical }\end{array}$ & Cardioembolic \\
\hline Hattingen et al., $2007^{2}$ & 33, female & $\begin{array}{l}\text { B/L body and anterior fornices; } \\
\text { genu of CC }\end{array}$ & AComm aneurysm clipping \\
\hline Adamovich et al., $2009^{7}$ & 53, female & B/L anterior fornices & Small vessel disease \\
\hline Murr et al., 2012 & 60, female & B/L anterior fornices & GCA \\
\hline
\end{tabular}

$\mathrm{B} / \mathrm{L}=$ bilateral; $\mathrm{CC}=$ corpus callosum; $\mathrm{AVM}=$ arteriovenous malformation; $\mathrm{AComm}=$ anterior communication artery; $\mathrm{GCA}=$ giant cell arteritis. 